\title{
Exome-wide association study reveals novel susceptibility genes to sporadic dilated cardiomyopathy
}

\section{Citation}

Esslinger, U., S. Garnier, A. Korniat, C. Proust, G. Kararigas, M. Müller-Nurasyid, J. Empana, et al. 2017. "Exome-wide association study reveals novel susceptibility genes to sporadic dilated cardiomyopathy." PLoS ONE 12 (3): e0172995. doi:10.1371/journal.pone.0172995. http:// dx.doi.org/10.1371/journal.pone.0172995.

\section{Published Version}

doi:10.1371/journal.pone.0172995

\section{Permanent link}

http://nrs.harvard.edu/urn-3:HUL.InstRepos:32630548

\section{Terms of Use}

This article was downloaded from Harvard University's DASH repository, and is made available under the terms and conditions applicable to Other Posted Material, as set forth at http:// nrs.harvard.edu/urn-3:HUL.InstRepos:dash.current.terms-of-use\#LAA

\section{Share Your Story}

The Harvard community has made this article openly available.

Please share how this access benefits you. Submit a story.

Accessibility 


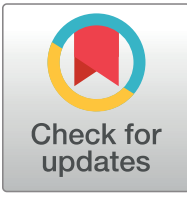

\section{OPEn ACCESS}

Citation: Esslinger U, Garnier S, Korniat A, Proust C, Kararigas G, Müller-Nurasyid M, et al. (2017) Exome-wide association study reveals novel susceptibility genes to sporadic dilated cardiomyopathy. PLoS ONE 12(3): e0172995. doi:10.1371/journal.pone.0172995

Editor: Amanda Ewart Toland, Ohio State University Wexner Medical Center, UNITED STATES

\section{Received: October 4, 2016}

Accepted: February 13, 2017

Published: March 15, 2017

Copyright: This is an open access article, free of all copyright, and may be freely reproduced, distributed, transmitted, modified, built upon, or otherwise used by anyone for any lawful purpose. The work is made available under the Creative Commons CCO public domain dedication.

Data Availability Statement: Genotyping data and phenotypes of the patients and controls are not available due to signed consent and ethical limitations. These data will not be available upon request. However, the summary statistics for association of all SNPs are included as supplementary files S5 and S6 Tables.

Funding: The study was supported by the Leducq Transatlantic Network: "Genomic, epigenomic and systems dissection of mechanisms underlying
RESEARCH ARTICLE

\section{Exome-wide association study reveals novel susceptibility genes to sporadic dilated cardiomyopathy}

\author{
Ulrike Esslinger ${ }^{1,2}$, Sophie Garnier ${ }^{1,2}$, Agathe Korniat ${ }^{1,2}$, Carole Proust ${ }^{1,2}$, \\ Georgios Kararigas $^{3}$, Martina Müller-Nurasyid ${ }^{4,5,6}$, Jean-Philippe Empana ${ }^{7,8}$, Michael \\ P. Morley ${ }^{9}$, Claire Perret ${ }^{1,2}$, Klaus Stark ${ }^{10}$, Alexander G. Bick ${ }^{11}$, Sanjay K. Prasad ${ }^{12}$, \\ Jennifer Kriebel ${ }^{13,14,15}$, Jin Li ${ }^{16}$, Laurence Tiret ${ }^{1,2}$, Konstantin Strauch ${ }^{4,17}$, Declan \\ P. O'Regan ${ }^{18}$, Kenneth B. Marguiles ${ }^{9}$, Jonathan G. Seidman ${ }^{11,19}$, Pierre Boutouyrie ${ }^{7,8,20}$, \\ Patrick Lacolley ${ }^{21}$, Xavier Jouven ${ }^{7,8,22}$, Christian Hengstenberg ${ }^{6,23}$, Michel Komajda ${ }^{1,2,24,25}$, \\ Hakon Hakonarson ${ }^{16}$, Richard Isnardd ${ }^{1,2,24,25}$, Eloisa Arbustini ${ }^{26}$, Harald Grallert ${ }^{13,14,15}$, Stuart \\ A. Cook ${ }^{27,28,29}$, Christine E. Seidman ${ }^{11,19}$, Vera Regitz-Zagrosek ${ }^{3}$, Thomas P. Cappola ${ }^{9}$, \\ Philippe Charron ${ }^{1,2,24,25,30}$, François Cambien ${ }^{1,2 *}$, Eric Villard ${ }^{1,2,24 *}$
}

1 Sorbonne Universités, UPMC Univ Paris 06, INSERM UMR-S1166, Team Genomics \& Pathophysiology of Cardiovascular Diseases, Paris, France, 2 ICAN Institute for Cardiometabolism and Nutrition, Paris, France, 3 Institute of Gender in Medicine and Center for Cardiovascular Research, Charite University Hospital, and DZHK, Berlin, Germany, 4 Institute of Genetic Epidemiology, Helmholtz Zentrum München-German Research Center for Environmental Health, Neuherberg, Germany, 5 Department of Medicine I, LudwigMaximilians-University Munich, Munich, Germany, 6 DZHK (German Centre for Cardiovascular Research), Partnersite Munich Heart Alliance, Munich, Germany, 7 INSERM, UMR-S970, Department of Epidemiology, Paris, France, 8 Université Paris Descartes, Sorbonne Paris Cité, Faculté de Médecine, Paris, France, 9 Penn Cardiovascular Institute and Department of Medicine, Perelman School of Medicine, University of Pennsylvania, Philadelphia, PA, United States of America, 10 Department of Genetic Epidemiology, University of Regensburg, Regensburg, Germany, 11 Department of Medecine and Genetics Harvard Medical School, Boston, MA, United States of America, 12 Royal Brompton Hospital, London, United Kingdom, 13 Research Unit of Molecular Epidemiology, Helmholtz Zentrum München-German Research Center for Environmental Health, Neuherberg, Germany, 14 Institute of Epidemiology II, Helmholtz Zentrum München-German Research Center for Environmental Health, Neuherberg, Germany, 15 German Center for Diabetes Research, Neuherberg, Germany, 16 Center for Applied Genomics, Children's Hospital of Philadelphia, Philadelphia, PA, United States of America, 17 Institute of Medical Informatics, Biometry and Epidemiology, Chair of Genetic Epidemiology, Ludwig-Maximilians-Universität, Munich, Germany, 18 Medical Research Council Clinical Sciences Centre, Faculty of Medicine, Imperial College London, London, United Kingdom, 19 Howard Hughes Medical Institute, Chevy Chase, MD, United States of America, 20 AP-HP, Georges Pompidou European Hospital, Pharmacology Department, Paris, France, 21 INSERM U1116, Université de Lorraine, Nancy, France, 22 AP-HP, Georges Pompidou European Hospital, Cardiology Department, Paris, France, 23 Deutsches Herzzentrum München, Technische Universität München, Munich, Germany, 24 AP-HP, Pitié-Salpêtrière Hospital, Cardiology Department, Paris, France, 25 AP-HP, Hôpital Pitié-Salpêtrière, Centre de Référence des Maladies Cardiaques Héréditaires, Paris, France, 26 IRCCS Fondazione Policlinico San Matteo, Pavia, Italy, 27 National Heart Centre Singapore, Singapore, 28 National Heart and Lung Institute, Imperial College London, London, United Kingdom, 29 Duke-NUS, Singapore, 30 Université de Versailles-Saint Quentin, AP-HP, Hôpital Ambroise Paré, Boulogne-Billancourt, France

* eric.villard@upmc.fr (EV); francois.cambien@upmc.fr (FC)

\section{Abstract}

\begin{abstract}
Aims
Dilated cardiomyopathy (DCM) is an important cause of heart failure with a strong familial component. We performed an exome-wide array-based association study (EWAS) to assess the contribution of missense variants to sporadic DCM.
\end{abstract}


dilated cardiomyopathy"; BestAgeing FP7 European Community project; and Conny Maeva Charitable Foundation. Other supports are from the British Heart Foundation, UK (PG/12/27/29489); NMRC Singapore; Medical Research Council, UK; Tanoto Foundation; and co-funded by the National Institute for Health Research (NIHR) Biomedical Research Centre based at Imperial College Healthcare NHS Trust, Biomedical Research Unit in Cardiovascular Disease at Royal Brompton \& Harefield NHS Foundation Trust and Imperial College London. The views expressed are those of the author(s) and not necessarily those of the NHS, the NIHR or the Department of Health. The PPS3 is organized under an agreement between INSERM and the IPC Center, and between INSERM and the Biological Research Center at the Européen Georges Pompidou hospital, Paris, France. We thank the "Caisse Nationale d'Assurance Maladie des Travailleurs Salariés". The PPS3 Study is funded by the National Research Agency (ANR), the Research Foundation for Hypertension (FRHTA), the Research Institute in Public Health (IRESP) and the Region lle de France (DIM). Genotyping in PPS3 substudy was supported by a grant from ANR (ANR-09-GENO-010). The KORA research platform was financed by the Helmholtz Zentrum München - German Research Center of Environmental Health, funded by the German Federal Ministry of Education and Research and by the State of Bavaria. Furthermore, KORA research was supported within the Munich Center of Health Sciences (MC Health), Ludwig-MaximiliansUniversität, as part of LMUinnovativ. The MAGNet consortium is funded by NIH R01HL105993 and NIH R01HL088577. Support for this work was provided in part by the Howard Hughes Medical Institute and National Institutes of Health. The funders had no role in study design, data collection and analysis, decision to publish, or preparation of the manuscript.

Competing interests: The authors have declared that no competing interests exist.

\section{Methods and results}

116,855 single nucleotide variants (SNVs) were analyzed in 2796 DCM patients and 6877 control subjects from 6 populations of European ancestry. We confirmed two previously identified associations with SNVs in BAG3 and ZBTB17 and discovered six novel DCMassociated loci (Q-value<0.01). The lead-SNVs at novel loci are common and located in TTN, SLC39A8, MLIP, FLNC, ALPK3 and FHOD3. In silico fine mapping identified HSPB7 as the most likely candidate at the ZBTB17 locus. Rare variant analysis (MAF $<0.01)$ demonstrated significant association for TTN variants only $(P=0.0085)$. All candidate genes but one (SLC39A8) exhibit preferential expression in striated muscle tissues and mutations in TTN, BAG3, FLNC and FHOD3 are known to cause familial cardiomyopathy. We also investigated a panel of 48 known cardiomyopathy genes. Collectively, rare $(n=228, P=0.0033)$ or common $(n=36, P=0.019)$ variants with elevated in silico severity scores were associated with DCM, indicating that the spectrum of genes contributing to sporadic DCM extends beyond those identified here.

\section{Conclusion}

We identified eight loci independently associated with sporadic DCM. The functions of the best candidate genes at these loci suggest that proteostasis regulation might play a role in DCM pathophysiology.

\section{Introduction}

Dilated cardiomyopathy (DCM) is a heart muscle disease characterized by left ventricular dilatation and systolic dysfunction in the absence of abnormal loading conditions or coronary artery disease (CAD). DCM is a major cause of sudden cardiac death and heart failure often requiring heart transplantation, its population-frequency is estimated to be around 1/500 [1]. Genetic studies of familial DCM have identified rare causal variants in more than 50 genes [2]. Genome-Wide Association Studies (GWAS) of sporadic DCM have revealed a few common variants associated with the disease [3]. A locus on chromosome 1 encompassing ZBTB17, $H S P B 7$ and CLCNKA, was replicated in several studies [3,4]. However, attempts to correlate the functions of these genes to DCM remained inconclusive[5]. In our prior GWAS, we also identified an association with a missense variant in $B A G 3$ and demonstrated the implication of BAG3 mutations in familial DCM [3].

Our prior GWAS had a limited sample size and used a genome-wide tagging array to estimate allele frequencies in stratified pools of DNA. We now report the results of an extended study in 6 populations of European ancestry, using the Illumina Human Exome Beadchip which mostly targets variants altering protein sequence (http://genome.sph.umich.edu/wiki/ Exome_Chip_Design). We report association analyses for single variants, candidate regions and a panel of 48 genes implicated in familial cardiomyopathy [2].To maximize power and because DCM is a relatively rare disease we conducted exome-wide genotyping in all available patients and controls instead of using a two-step discovery/replication design.

\section{Methods}

See S1 Methods for details, in brief: 


\section{Ethics statement}

Written informed consent was obtained from all study participants. All samples were collected in accordance with the Helsinki declaration and study protocols were approved by the ethics committees of the participating centers: UK population: Southampton and south west Hampshire research ethics committee (09/H0504/104); USA population 1: The Partners Human Research Committee, IRB of Partners Healthcare, Brigham and Women's Hospital; USA population 2: MAGNet study-University of Pennsylvania Institutional Review Board; Eurogene Ethics: CPP comité de protection des personnes dans la recherche biomédicale, Faculty hospital Pitié-Salpêtrière, Paris (ref 66-01); Cardigene Ethics: CPP comité de protection des personnes dans la recherche biomédicale, Faculty hospital Pitié-Salpêtrière, Paris; PHRC Ethics: $\mathrm{CPP}$ comité de protection des personnes dans la recherche biomédicale, Faculty hospital PitiéSalpêtrière, Paris (ref 63-05); German population- Charite University Hospital Ethics Committee, Berlin, Germany.

\section{Populations included and samples collection}

All subject included in the study gave informed consent; the research protocol was approved by local ethic committees and complies with the Declaration of Helsinki. Patients with sporadic idiopathic DCM and controls from six populations of European descent, recruited in Germany, France, UK, USA and Italy, were included in this EWAS. Sporadic DCM was diagnosed according to standard criteria and known secondary causes of the disease as well as familial cases (when relevant information was available) were excluded. Control subjects from the same country of origin were selected for each group of patients except for the MAGNet Study patients (USA2 population) for whom a control group was artificially defined by subsampling 1,000 individuals from the German control group (see S1 Methods-Variant-level analysis).

\section{Genotyping and data preprocessing}

Genotyping was done with Illumina HumanExome BeadChips using a standard protocol. Quality control was performed with the 1.9 version of the PLINK software [6] and in the $R$ version 3.1 environment. Markers with genotyping success rate $<99 \%$ and samples with $<99 \%$ of markers available were excluded.

\section{Protein interaction study}

HEK293 cells were transfected with vectors encoding for GFP-tagged BAG3 or GFP (as a negative control). $48 \mathrm{~h}$ post-transfection cell lysates were subjected to 2 independent protein interaction analyses. For GST pull-down experiments, HEK293 cells were transfected with vectors expressing GFP alone or GFP-tagged BAG3 proteins. Total cell lysates were incubated with Glutathione Sepharose beads complexed with GST-HspB7 recombinant proteins. GST tagged HspB7 proteins were purified from a bacterial expression system (BL21 E. coli). For co-immunoprecipitation, HEK293 cells were co-transfected with Flag-HSPB7 and GFP or GFP-BAG3 vectors. Total cell lysates were incubated with magnetic Protein A Dynabeads and subjected to immunoprecipitation using an anti-GFP antibody. Input fractions, GST-pull down and GFPcoimmunoprecipitated proteins, were revealed by Western-Blot.

\section{Statistical analysis}

All statistical models were adjusted on age and gender and on the 20 first principal components (PCs) estimated from the genetic relatedness matrix (GRM) [7] to account for a possible population stratification. 


\section{Variant-level analysis}

Association between case-control status and each variant was assessed using logistic regression, variant effect being modeled either as additive or dominant in PLINK. Homogeneity of effects of DCM-associated variants across populations was tested by a meta-analysis of population-specific data. To account for false discovery rate, a Q-value $(R / Q$ value package) threshold of 0.01 was chosen. S1A Table reports the power of our study for various MAF and allelic risk.

\section{Region and gene set-level analyses}

We used SKAT [8] to evaluate the global contribution of sets of variants. The analysis was performed for rare $(\mathrm{MAF}<0.01)$, common, and all variants combined. Regional analyses were centered on the candidate regions discovered in the variant-level analyses. For the gene-set analysis, genes known to be implicated in familial cardiomyopathy were identified [2] and all available variants on these genes were tested both at the gene level and as a whole. S1B Table reports the power of our study for the gene set analyzes.

\section{Fine mapping by imputing variants in the regions of interest}

To identify non-genotyped variants in strong linkage disequilibrium (LD) with the lead-SNVs, imputation was performed across regions encompassing the loci identified in the variant-level analysis [9].

\section{Bioinformatics resources}

Variant functionality was assessed using the Combined Annotation Dependent Depletion $(C A D D)$ framework [10]. We also determined tissue-specific gene expression in silico using the Genotype-Tissue Expression (GTEx) database [11].

\section{Results}

The study included 2796 DCM patients (643 females) and 6877 control subjects ( 3045 females) (S2 Table). After exclusion of variants that were monomorphic in cases and controls, 95499 SNVs were available for analysis. At 1\% Q-value, 11 SNVs at 8 distinct loci are significantly associated to DCM (Fig 1, Table 1). Re-analysis of the data after removal of the 85 cases with identified familial DCM forms did not modify the results (S7 Table). Two of these loci were previously identified (ZBTB17-HSPB7 and BAG3) and six are novel (TTN, SLC39A8, MLIP, FLNC, NMB-ALPK3, FHOD3). The QQ-plot of association statistics (S1 Fig) shows that population stratification was apparently well-controlled (lambda $=0.991$ ). Population-specific Manhattan and QQ-plots are separately reported in S2 Fig. Based on the QQ-plot and lambda value (0.969) for the USA2 cohort, the reconstructed control group for the MAGNet DCM cases appears appropriate. For all loci, the effect of the lead SNP is quite homogeneous across populations (Fig 2). Ten of the 11 SNVs encode missense residues, as expected from the enrichment of the exome array for this category of variants.

\section{ZBTB17-HSPB7 locus}

The lead SNV, $r s 10927875$ (ZBTB17, c. $-3+222 \mathrm{G}>\mathrm{A}, \mathrm{MAF}=0.31$ ), is located in an intron of ZBTB17. As observed in our earlier GWAS [3], this SNP confers a reduced risk of DCM $\left(\mathrm{OR}=0.77(0.71-0.83), \mathrm{P}=8.1 \times 10^{-13}\right)$. Imputation revealed a large number of SNVs in strong LD with $r s 10927875$ (S3A Table), but as a result of the "Yin-Yang" haplotypic structure of the region we could not determine the most likely causal variant or gene (Fig $3 A$ ). However, several imputed SNVs located within or downstream the heat shock $27 \mathrm{kDa}$ protein family, 


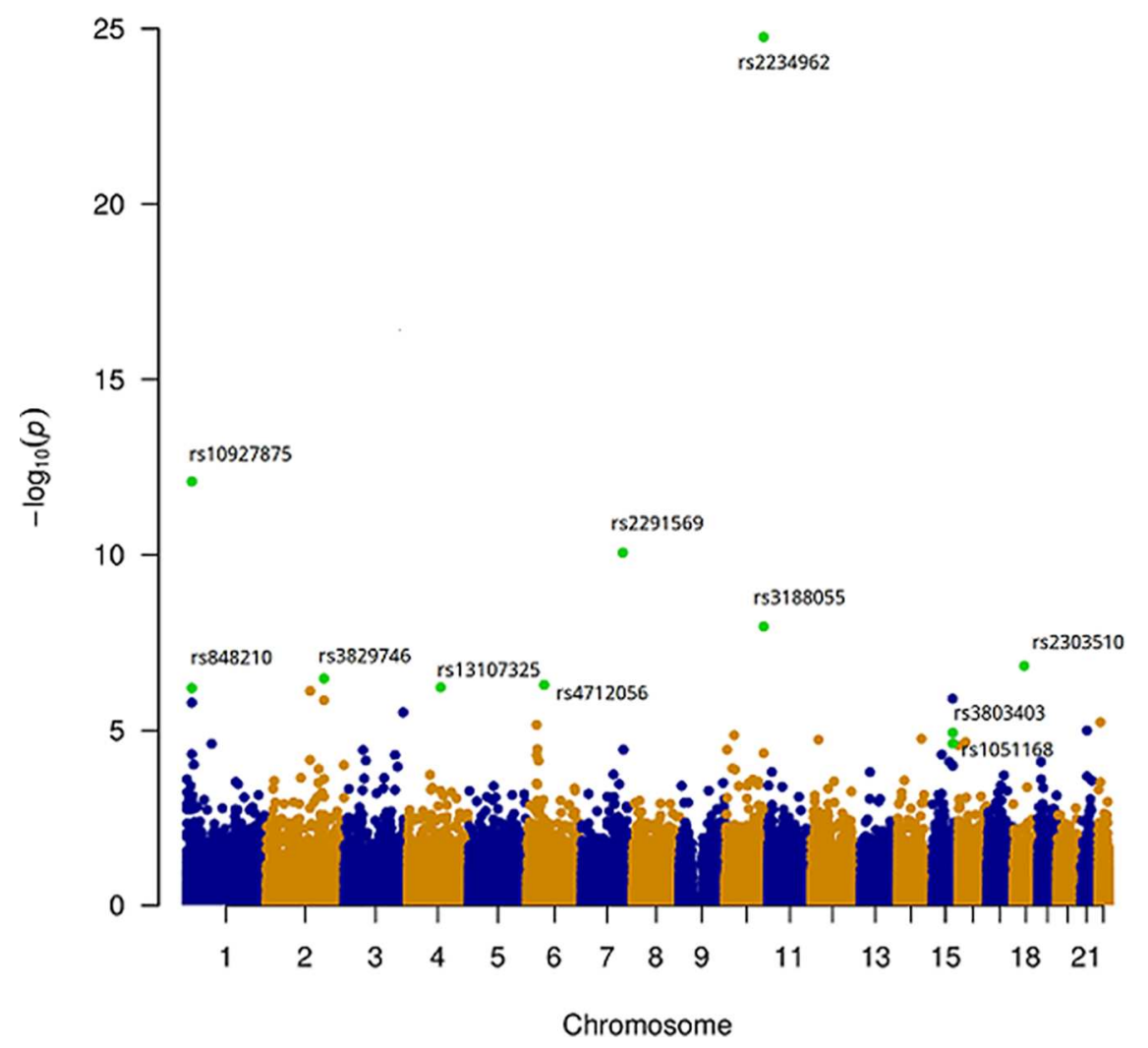

Fig 1. Manhattan Plot of association P-values. 95,499 variants were investigated for association with DCM by logistic regression analysis. Associations are summarized in a Manhattan plot (R/qqman package) which displays the eleven SNVs significantly associated with DCM $(Q$-values $<0.01)$ as green dots. Note that the applied logistic model assumed an additive mode of inheritance. For variants on chromosome 15 in the $A L P K 3$ region, a dominant mode of inheritance was better supported by the data (see Table 1 for corresponding P-values)

doi:10.1371/journal.pone.0172995.g001

member 7 gene (HSPB7) had slightly more significant P-values than rs10927875 (S3A Table). Generally, SNVs within $H S B P 7$ had more deleterious CADD scores than those within ZBTB17 or CLCNKA (another candidate within the region [4]). In addition, GTEx analysis showed that $H S P B 7$ is mostly expressed in the heart and skeletal muscles. Using GST pull-down experiment and co-immunoprecipitation we also observed that recombinant BAG3 interacts with HSPB7 (Fig 4). Overall, this suggests that $H S P B 7$ is the best candidate to explain the association of variants in this region with DCM.

\section{TTN locus}

Titin is a major component of the sarcomere and an important familial DCM gene [12]. The lead SNV, $r s 3829746$ in $(T T N, c .56704 \mathrm{~A}>\mathrm{G}$, p.Ile18902Val, MAF $=0.23)$ confers a reduced risk of DCM $\left(\mathrm{OR}=0.81(0.75-0.88), \mathrm{P}=3.4 \times 10^{-7}\right)$. A regional plot of all variants (genotyped and imputed) shows that several are in tight LD with the lead SNV (Fig 3B) and associated with DCM. Among these SNVs, $r s 2042996$ (p.Thr21403Ile) has the highest CADD score (20.1) (S3B Table). According to GTEx rs3829746 is associated with reduced TTN expression in the left ventricle $(\mathrm{P}=0.04)$ and atrial appendage $(\mathrm{P}=0.006)$. Unlike TTN truncating variants that cause DCM, the DCM-associated TTN missense variants present on the exome-array are independent of TTN exon usage. (S1 Methods-Exon usage in TTN). 
Table 1. Variants associated with DCM.

\begin{tabular}{l|l|l|l|l|l|l|l}
\hline & MAF & OR $(95 \%$ Cl) & P-value & Q-value & P-Het & Gene & \multicolumn{1}{|c|}{ AA Change } \\
\hline rs848210 & A: $0.45(0.39-0.49)$ & $1.182(1.11-1.26)$ & $6.3 \times 10^{-07}$ & $6.0 \times 10^{-03}$ & 0.5 & SPEN & N/D \\
\hline rs10927875 & A: $0.31(0.26-0.37)$ & $0.768(0.71-0.83)$ & $8.1 \times 10^{-13}$ & $3.9 \times 10^{-08}$ & 0.83 & ZBTB17 & - \\
\hline rs3829746 & G: $0.23(0.21-0.24)$ & $0.810(0.75-0.88)$ & $3.4 \times 10^{-07}$ & $4.6 \times 10^{-03}$ & 0.039 & TTN \\
\hline rs13107325 & A: $0.08(0.07-0.12)$ & $1.348(1.20-1.52)$ & $6.0 \times 10^{-07}$ & $6.0 \times 10^{-03}$ & 0.61 & SLC39A8 & A/T \\
\hline rs4712056 & G: $0.35(0.34-0.36)$ & $1.191(1.11-1.28)$ & $5.1 \times 10^{-07}$ & $6.0 \times 10^{-03}$ & 0.53 & MLIP & V/I \\
\hline rs2291569 & A: $0.08(0.05-0.09)$ & $0.651(0.57-0.74)$ & $8.7 \times 10^{-11}$ & $2.8 \times 10^{-06}$ & 0.27 & FLNC & R/Q \\
\hline rs2234962 & G: $0.19(0.15-0.22)$ & $0.620(0.57-0.68)$ & $1.7 \times 10^{-25}$ & $1.6 \times 10^{-20}$ & 0.14 & BAG3 & C/R \\
\hline rs3188055 & G: $0.34(0.33-0.35)$ & $1.223(1.14-1.31)$ & $1.1 \times 10^{-08}$ & $2.6 \times 10^{-04}$ & 0.84 & INPP5F & N/D \\
\hline rs1051168 & A: $0.30(0.27-0.35)$ & $1.273(1.16-1.40)$ & $4.1 \times 10^{-07}$ & $4.9 \times 10^{-03}$ & 0.32 & NMB & P/T \\
\hline rs3803403 & G: $0.30(0.28-0.35)$ & $1.276(1.16-1.40)$ & $2.9 \times 10^{-07}$ & $4.0 \times 10^{-03}$ & 0.29 & ALPK3 & T/S \\
\hline rs2303510 & A: $0.31(0.29-0.34)$ & $0.824(0.77-0.89)$ & $1.5 \times 10^{-07}$ & $2.3 \times 10^{-03}$ & 0.023 & FHOD3 & V/I \\
\hline
\end{tabular}

For rs1051168 (NMB) and rs3803403 (ALPK3) the result of the dominant model is shown as it is better supported than the additive test which is presented for all other variants. The minor frequency allele is the effect allele.

MAF: Minor allele frequencies (ranges across 6 populations).

OR: Odds-Ratio estimated using a logistic model adjusted on gender and first $20 \mathrm{PCs}$.

Q-value threshold (0.01)

Het: test of homogeneity of effects across populations.

SPEN: spen family transcriptional repressor; ZBTB17: zinc finger and BTB domain containing 17; TTN: titin; SLC39A8: solute carrier family 39 (zinc transporter), member 8; MLIP: muscular LMNA-interacting protein; FLNC: filamin C, gamma; BAG3: BCL2-associated athanogene 3; INPP5F: inositol polyphosphate-5-phosphatase F; NMB: neuromedin B; $A L P K 3$ : alpha-kinase 3; FHOD3: formin homology 2 domain containing 3.

\section{SLC39A8 locus}

Solute carrier family 39 member 8 gene encodes encodes a transmembrane metal-ion transporter exhibiting highly pleiotropic effects. The lead SNV rs13107325 (SLC39A8, c.1171G >A, p.Ala391 Thr, MAF $=0.08)$ confers an increased risk of DCM $\left(\mathrm{OR}=1.35(1.20-1.52), \mathrm{P}=6.0 \times 10^{-7}\right)$. Imputation reveals little genetic variability at this locus (Fig 3C, S3C Table) and rs13107325 has the highest $C A D D$ score (35.0), suggesting that it might be the causal variant. The possible implication of this locus in DCM is intriguing, given that SLC39A8 is minimally expressed in the heart.

\section{MLIP locus}

Muscular Lamin A (LMNA)-interacting protein interacts with LMNA, a structural component of nuclear lamina known to be implicated in familial DCM [13]. The lead SNV rs4712056 $(M L I P$, c.475G $>$ A, p.Val159Ile, MAF $=0.35)$ is associated with an increased risk of DCM $\left(\mathrm{OR}=1.19(1.11-1.28), \mathrm{P}=5.1 \times 10^{-7}\right)$. Several imputed variants in the gene are more strongly associated with DCM than rs4712056 (Fig 3D, S3D Table). All of them are intronic or located upstream of the sequence encoding the short cardiac transcript of MLIP. The strongest association implicates $r s 35182047$, a small intronic insertion (c.64-12401_64-12400insAT). Although the $C A D D$ score of this variant is modest (2.33), its DCM-associated risk (OR $=1.30$ (1.20$\left.1.40, \mathrm{P}=2.5 \times 10^{-10}\right)$ is substantially higher than that of $r s 4712056$.

\section{FLNC locus}

Filamin $\mathrm{C}$ is an actin-crosslinking protein, specifically expressed in cardiac and skeletal muscles. The lead SNV rs2291569 (FLNC, c.4700G >A, p.Arg1567Gln, MAF $=0.09$ ) is associated with a reduced risk of DCM $\left(\mathrm{OR}=0.65(0.57-0.74), \mathrm{P}=8.7 \times 10^{-11}\right)$. Fine mapping analysis 
rs10927875:A - ZBTB17

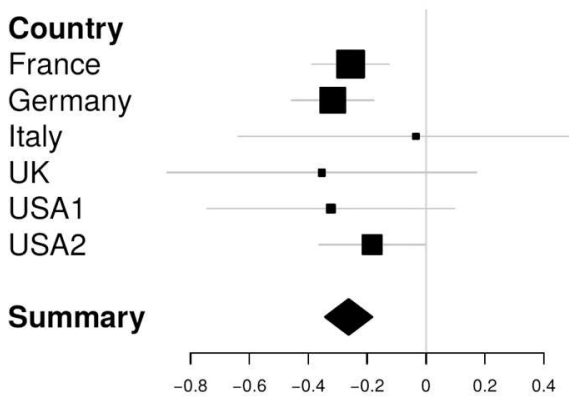

rs13107325:A - SLC39A8

\section{Country}

France

Germany

Italy

UK

USA1

USA2

Summary

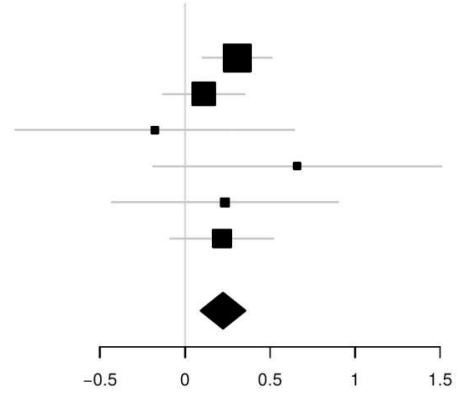

$$
\text { rs2291569:A - FLNC }
$$

\section{Country}

France

Germany

Italy

UK

USA1

USA2

Summary

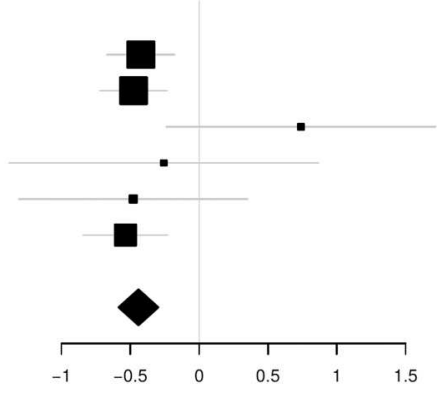

rs3803403:G - ALPK3

\section{Country}

France

Germany

Italy

UK

USA1

USA2

Summary

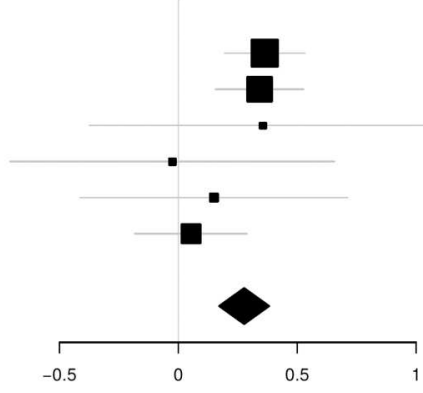

rs3829746:G - TTN

\section{Country}

France

Germany

Italy

UK

USA1

USA2

Summary

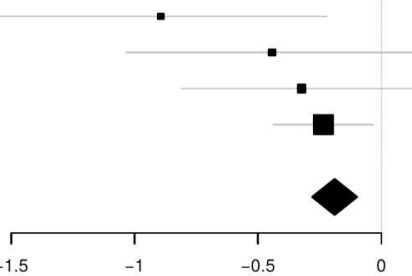

rs4712056:G - MLIP

\section{Country}

France

Germany

Italy

UK

USA1

USA2

Summary

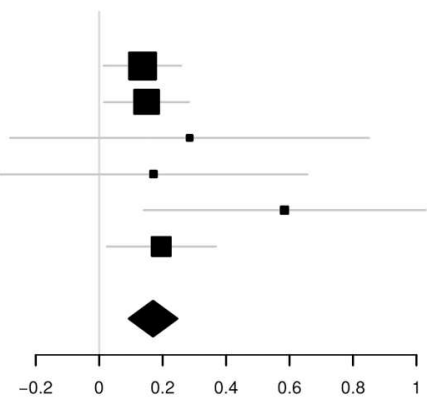

rs2234962:G - BAG3

\section{Country}

France

Germany

Italy

UK

USA1

USA2

Summary

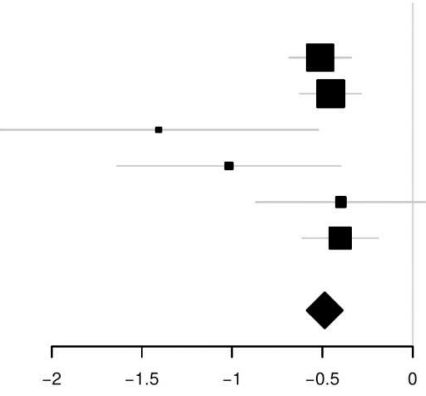

rs2303510:A - FHOD3

\section{Country}

France

Germany

Italy

UK

USA1

USA2

Summary

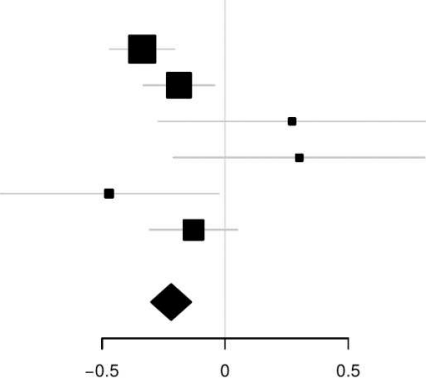

Fig 2. Forest Plots of odd-ratios (log) in the different populations. The results show that the associations were largely homogeneous across populations (See also heterogeneity column in Table 2)

doi:10.1371/journal.pone.0172995.g002 

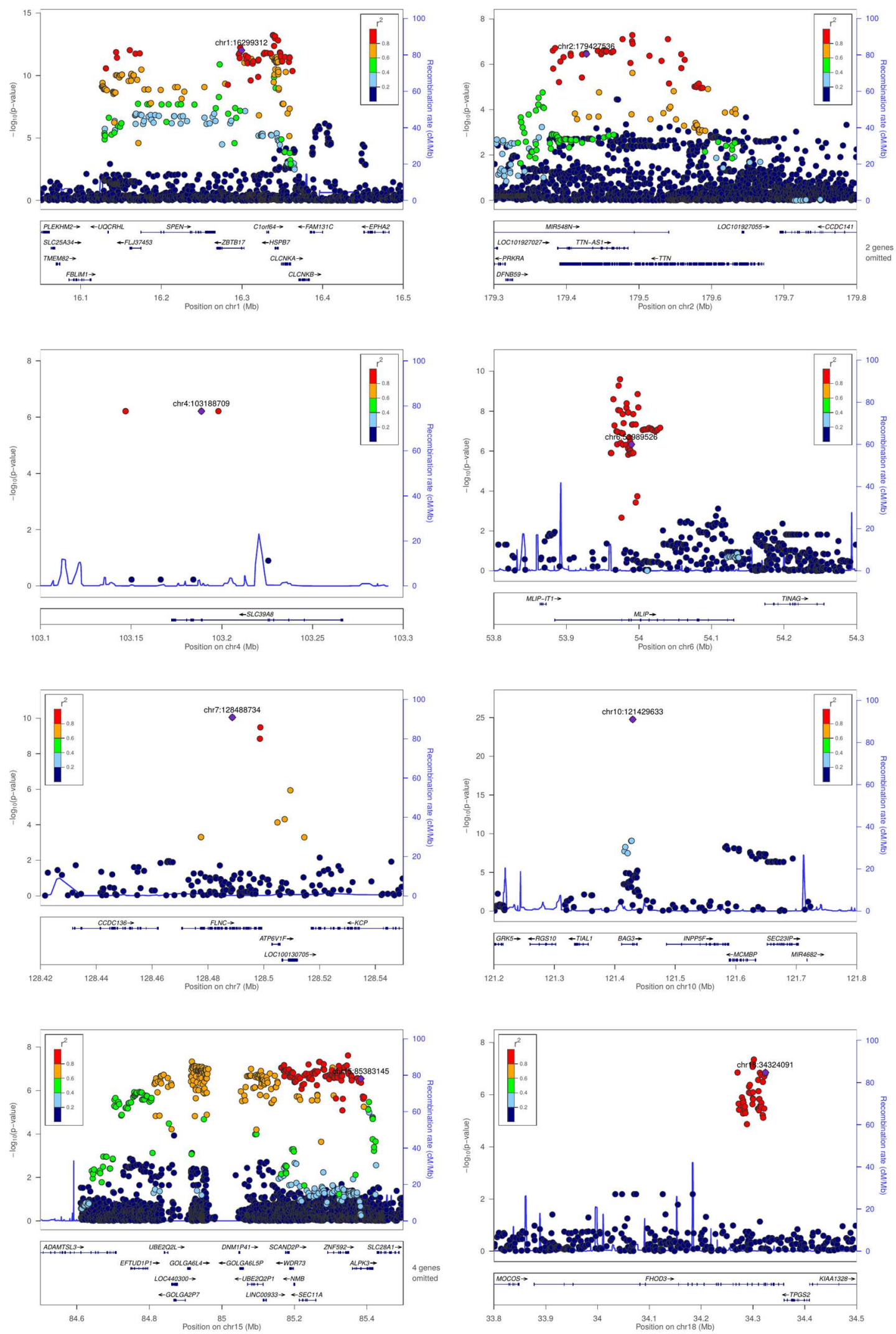
Fig 3. Regional plots of association at 8 loci. P-values, obtained from logistic regression analysis of genotyped and imputed variants in genomic regions demonstrating significant association with DCM are depicted. In each region, the genotyped lead-SNV is identified by its position and the other variants are colored to reflect their LD with the lead-SNV. doi:10.1371/journal.pone.0172995.g003

shows that two imputed variants located in the 3'UTR of FLNC are in strong LD with rs22915 69 and exhibit similar ORs (Fig 3E, S3E Table).

\section{BAG3 locus}

This locus was the second one identified in our earlier GWAS.(3) $r s 2234962(B A G 3, c .451 \mathrm{~T}>\mathrm{C}, \mathrm{p}$. Cys151Arg, MAF $=0.19)$ confers a reduced risk of DCM $\left(\mathrm{OR}=0.62(0.57-0.68), \mathrm{P}=1.7 \times 10^{-25}\right)$. A nearby SNV, $r s 3188055$, located in the INPP5F is no longer significant after conditioning on $r s 2234962$. When also considering imputed variants at the locus, $r s 2234962$ is by far the most significant and it also has the highest CADD score (24.2) (Fig 3F, S3F Table).

\section{NMB-ALPK3 locus}

Two missense variants in strong $\mathrm{LD}\left(\mathrm{r}^{2}=0.82\right), r s 1051168$ in Neuromedin $\mathrm{B}(N M B, \mathrm{c} .217 \mathrm{C}>\mathrm{A}$, p.Pro73Thr, MAF $=0.30)$ and $r s 3803403$ in alpha-kinase $3(A L P K 3, c .1241 \mathrm{C}>\mathrm{G}$, p.Thr414Ser, MAF $=0.30)$ are associated with DCM at this locus. The minor allele at both loci exerts a dominant effect on DCM risk ( $\mathrm{OR}=1.27$ [1.16-1.40]). Fine-mapping analysis identifies a single major haplotypic structure encompassing NMB, ALPK3 and several other genes (Fig 3G). $C A D D$ scores do not orient towards causal variants among these tightly associated SNVs ( $33 G$ Table). However, GTEx indicates that the genes in the interval are not or very lowly expressed in the heart or skeletal muscle, except $A L P K 3$ which is almost exclusively expressed in these tissues. ALPK3 encodes a nuclear kinase implicated in the differentiation of cardiomyocyte and Alpk3-deficient mice develop cardiomyopathy [14].

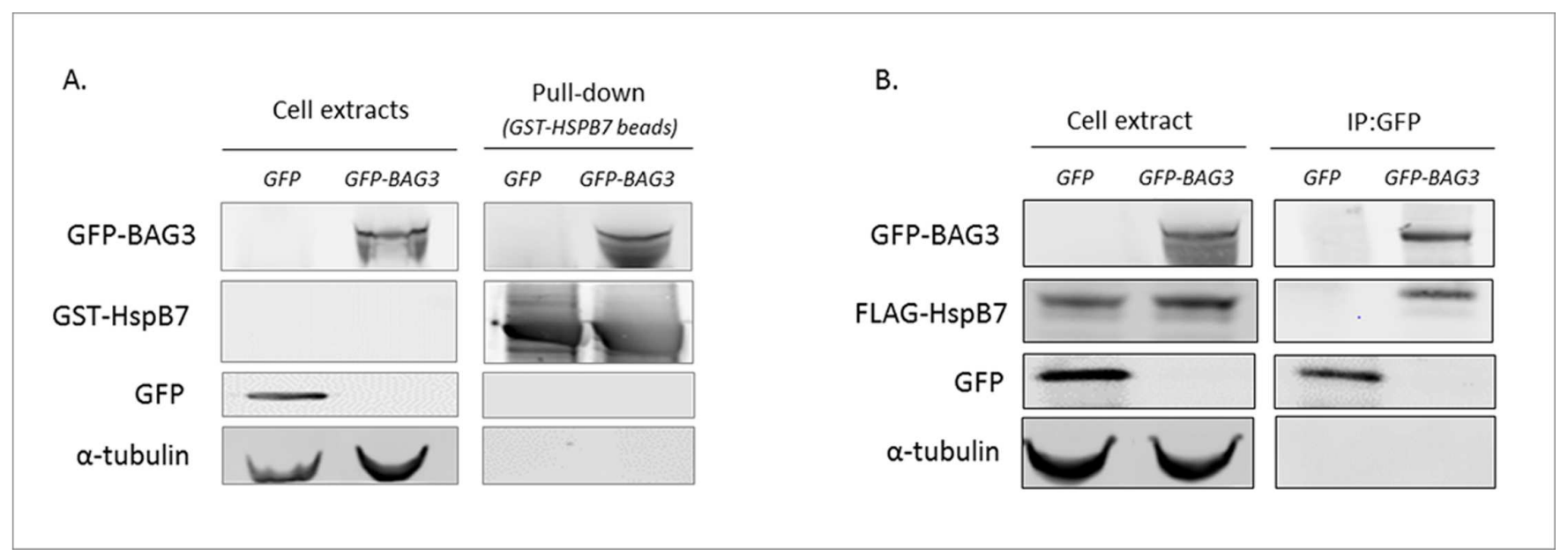

Fig 4. BAG3 interacts with HspB7. (A) GST Pull-Down showing interaction of GFP-BAG3 expressed in HEK293 cells and recombinant GST-HSPB7. GFP-BAG3 was expressed in HEK293 cells (cell extract panel) and and GST-HSPB7 was produced in a bacterial expression system. GST-HSPB7 co-sediment with GFP-BAG3 but not with GFP alone indicating specific BAG3/GST-HSPB7 interaction (Pull-Down panel). (B) Coimmunoprecipitation experiment showing interaction of Flag-HSPB7 and GFP-BAG3 in HEK293 cells. GFP alone or GFP-BAG3 were co-expressed together with Flag-HSPB7 in HEK293 cells (cell extract panel) and subjected to immunoprecipitation with an antibody against GFP. Only GFP-BAG3 immunoprecipitated with FLAG-HSPB7 (IP:GFP panel). Western blottings in (A) and (B) used HSPB7 (for GST-HSPB7), GFP (for GFP and GFP-BAG3), and $\alpha$-tubulin specific antibodies.

doi:10.1371/journal.pone.0172995.g004 


\section{FHOD3 locus}

Formin homology 2 domain containing 3 regulates actin assembly and sarcomere organization in striated muscles. The lead SNV rs2303510(FHOD3, NM_025135.4:c.3591G>A, NP_079411.2:p.Val1151 >Ile, MAF $=0.31)$ is associated with a reduced risk of DCM $\left(\mathrm{OR}=0.82(0.77-0.89), \mathrm{P}=1.5 \times 10^{-07}\right)$. Imputation identifies several SNVs, most of them intronic, in strong LD with $r$ s2303510 and clustered within a relatively narrow region of the FHOD3 sequence (Fig 3H, S3H Table). Among these SNVs, the highest CADD score (22.8) is observed for the missense rs2303510 variant.

\section{Tissue expression of identified candidate genes}

According to GTEx, with the exception of SLC39A8, the best candidate genes at the DCMassociated loci are all preferentially expressed in heart or skeletal muscle tissues. However, except for TTN, their expression levels in these tissues are unaltered by the lead-SNVs.

\section{Gene-level analysis}

Because analysis of single SNVs lacks power for detecting association with rare variants, we tested whether genotyped variants in the candidate regions were collectively associated with DCM. A first analysis showed a significant association of rare variants $(\mathrm{MAF}<0.01)$ at the ZBTB17 and TTN loci with DCM (Table 2). However, when conditioning on the lead SNP at each locus, only the association of TTN rare variants remained significant $(\mathrm{P}=0.013)$. When including all variants (rare and common) in the analysis and conditioning on the lead SNVs, there was still a significant association $(\mathrm{P}<0.05)$ at the ZBTB17, TTN and BAG3 loci which implies residual associations independent of the lead-SNVs (Table 2).

\section{Familial DCM gene-set analysis}

Sixty genes are reported to be implicated in familial cardiomyopathies [2]. To investigate whether these genes might also have a role in sporadic DCM, we tested their association at the gene level. After discarding BAG3 and TTN and genes harboring no variant in our data set, 48 genes and 608 variants (478 of which are rare) were tested (S4 Table). No association was observed for rare variants after Bonferroni correction for 48 genes. For common variants, the only significant association was observed for MYBPC3 (4 variants, $\mathrm{P}=5.7 \times 10^{-5}, \mathrm{P}<0.003$ after Bonferroni correction). In the MYBPC3 gene, the most significant SNV had a P-value of

Table 2. Gene-level association analysis of genes identified in the SNP-level analysis

\begin{tabular}{|c|c|c|c|c|}
\hline Region & $\begin{array}{l}\text { number of variant (rare } \\
\qquad \mathrm{f}<0.01 \text { ) }\end{array}$ & $\begin{array}{l}\text { All variants P-value } \\
\text { (adjusted }^{\$} \text { ) }\end{array}$ & $\begin{array}{l}\text { Rare variants P-value } \\
\text { (adjusted }^{\$} \text { ) }\end{array}$ & $\begin{array}{l}\text { Common variants P-value } \\
\text { (adjusted }^{\$} \text { ) }\end{array}$ \\
\hline ZBTB17 & $54(47)$ & $1.1 \times 10^{-12}(0.007)$ & $0.046(0.4)$ & $2.14 \times 10^{-12}(0.0027)$ \\
\hline TTN & $457(369)$ & $3.1 \times 10^{-06}(0.0083)$ & $0.0085(0.013)$ & $1.20 \times 10^{-05}(0.048)$ \\
\hline SLC39A8 & $4(2)$ & $9.5 \times 10^{-05}(0.23)$ & $0.13(0.12)$ & $1.65 \times 10^{-05}(0.5)$ \\
\hline MLIP & $24(15)$ & $1.2 \times 10^{-04}(0.19)$ & $0.91(0.81)$ & $1.68 \times 10^{-06}(0.049)$ \\
\hline FLNC & $72(61)$ & $2.3 \times 10^{-04}(0.46)$ & $0.32(0.4)$ & $2.34 \times 10^{-05}(0.48)$ \\
\hline BAG3 & $32(22)$ & $2.6 \times 10^{-20}(0.037)$ & $0.23(0.53)$ & $4.36 \times 10^{-23}(0.0094)$ \\
\hline ALPK3 & $42(33)$ & $2.5 \times 10^{-02}(0.8)$ & $0.55(0.53)$ & $3.71 \times 10^{-03}(0.89)$ \\
\hline FHOD3 & $40(30)$ & $6.9 \times 10^{-04}(0.27)$ & $0.083(0.086)$ & $7.80 \times 10^{-04}(0.77)$ \\
\hline
\end{tabular}

The gene-level analysis was performed using the R/SKAT_CommonRare function (See S1 Methods).

$\$$ P-values adjusted on lead-SNV.

doi:10.1371/journal.pone.0172995.t002 
$3.06 \times 10^{-03}$. The entire set of 608 variants was associated with DCM $(\mathrm{P}=0.0067)$ and the association was strengthened for variants with a $C A D D$ severity score $>20(\mathrm{n}=264, \mathrm{P}=0.0005)$; both rare $(\mathrm{P}=0.0033)$ and common variants $(\mathrm{P}=0.019)$ contributed to this association (Table 3).

\section{Discussion}

In this EWAS of sporadic DCM we confirmed associations with variants in ZBTB17-HSPB7 and $B A G 3$ and identified six novel loci. Statistical analyses, cardiac tissue expression, and physiology suggest that the most likely causal genes are HSPB7, BAG3, TTN, SLC39A8, MLIP, FLNC, ALPK3 and FHOD3.

Our data provide evidence that non-coding variants close to or within $H S P B 7$ are more likely to account for the observed association at the ZBTB17 locus. The genetic mechanism linking the risk haplotype to HSPB7 functional modulation in absence of detectable eQTL is unknown. HSPB7 (commonly referred as cardiovascular Heat Shock Protein; cvHSP) is a member of the small HSPB family of molecular chaperones. It is a potent polyglutamin aggregation suppressor that assists the loading of misfolded proteins or small protein aggregates into autophagosomes [15]. In addition, our in vitro experiments demonstrate a physical interaction of BAG3 with HSPB7 (Fig 4) suggesting functional relationships between the 2 proteins that may be relevant for their genetic implication in DCM pathophysiology. The strongest association with sporadic DCM in our EWAS involved rs2234962 which encodes a p.Cys151Arg substitution in BAG3. The interaction signal of BAG3 Arg151 and Cys151 isoforms with HSPB7 was similar (data not shown), suggesting no direct effect of the polymorphism on HSPB7 binding The p.Cys151Arg variant is located between two conserved Ile-Pro-Val (IPV) motifs involved in BAG3 complex formation with HSPB6 and HSPB8. Interestingly, a p.Pro209Leu mutation responsible for myofibrillar myopathy associated with cardiomyopathy is located in one of the two IPV motifs [16]. Whether p.Cys151 Arg modifies the interaction of BAG3 with HSPBs partners and affects the functional potential of the complex is currently unknown.

Common haplotype-tagging variants of the titin gene were associated with small differences in DCM risk in this EWAS. This extends the spectrum of TTN genetic variants that affect DCM risk, from highly penetrant mutations responsible for familial DCM [12] to common haplotypes with low penetrance associated with sporadic DCM. A potential consequence of common DCM-associated TTN variants, in line with the pathogenic mechanisms suggested by the candidate genes identified in this EWAS, is the proteotoxic effect of accumulating truncated or aggregate prone mutant TTN in cardiomyocytes.

The rs13107325 SNV in SLC39A8 has been shown in GWAS to be associated with several traits affecting cardiovascular risk, including blood pressure. It is therefore conceivable that it has systemic consequences that raise the risk of DCM but were not considered in our disease exclusion criteria. Because SLC39A8 encodes a zinc transporter [17], its association with DCM may also be related to the cardioprotective role of zinc [18].

Table 3. DCM gene set association analysis

\begin{tabular}{l|c|c|c|c}
\hline variants subset & Number of variants (rare f<0.01) & All variants P-value & Rare variants P-value & Common variants P-value \\
\hline All & $608(478)$ & 0.0067 & 0.013 & 0.091 \\
\hline CADD20 & $264(228)$ & 0.0005 & 0.0033 & 0.019 \\
\hline CADD25 & $71(63)$ & 0.016 & 0.047 & 0.064 \\
\hline CADD30 & $30(26)$ & 0.064 & 0.19 & 0.07 \\
\hline
\end{tabular}

All variants on 48 familial DCM genes (excluding BAG3 and TTM) also categorized by $C A D D$ severity scores.

doi:10.1371/journal.pone.0172995.t003 
In the nuclear envelope, MLIP (also known as CIP) directly interacts with the $\mathrm{N}$-terminal region of lamin (LMNA) [19]. Dominant mutations in LMNA cause DCM and other hereditary multisystemic diseases and several pathogenic mutations of LMNA are located in its MLIP interacting domain [20]. In mice, Mlip interacts with Isl1, a transcription factor required for cardiomyocyte differentiation, and represses its transcriptional activity [21]. Notably, the DCM-associated SNV ( $r s 4712056$, p.Val159Ile) is located within the Isl1-interacting region of MLIP. MLIP has recently been shown to be a key regulator of cardiomyopathy that has potential as a therapeutic target to attenuate heart failure progression [22].

Filamin $\mathrm{C}$ is involved in the organization of actin filaments, it serves as a scaffold for signaling proteins and interacts with several Z-disk proteins. FLNC mutations in humans and mice cause hypertrophic cardiomyopathy [23] and myofibrillar myopathy, a form of muscular dystrophy with concurrent cardiomyopathy [24]. These pathologies are characterized by myofibrillar disorganization, accumulation of myofibrillar degradation products and ectopic expression of multiple proteins [25]. FLNC mutations induce massive protein aggregates within skeletal muscle fibers and altered expression of chaperone proteins and components of proteasomal and autophagic degradation pathways. Interestingly, functional interaction between FLNC and HSPB7 or BAG3, two genes confirmed by this study, have been previously reported [26,27]. In addition to the fact that $B A G 3$ mutations also causes myofibrillar myopathy it suggests the hypothesis that dysregulation of proteostasis could be a common mechanism underlying myofibrillar myopathy and DCM.

Cardiac FHOD3 plays a crucial role in the sarcomere organization of cardiomyocytes, is essential for heart myofibrillogenesis [28] and is required for the maintenance of the contractile structures in heart muscle. A cardiac isoform of FHOD3 is targeted to thin actin filaments via phosphorylation of tyrosine residue preventing autophagy dependent degradation [29]. Most DCM-associated SNVs in our EWAS are clustered in a region in 3' of FHOD3 that encodes the Formin $\mathrm{FH} 2$ domain of the protein, which is implicated in actin polymerization [30]. A FHOD3 variant, Y1249N, has been reported in a Japanese patient with a dominant form of DCM. In vivo functional analysis showed that this variant may impair actin filament assembly, thus providing some support for the implication of FHOD3 in the pathogenesis of DCM [31].

Alpha-kinase 3 ( $A L P K 3 / M I D O R I)$ was initially described as a myocyte-specific gene that promotes differentiation of P19CL6 cells into cardiomyocytes [32]. The pattern of expression of $A L P K 3$ in differentiating cardiomyocytes nucleus is similar to that of transcription factors specific of the cardiogenic lineage [33] but its function is still largely unknown. Recently recessive mutations in ALPK3 have been reported to cause pediatric DCM [34].

In addition to TTN and BAG3, MYBPC3 was present in the cardiomyopathy gene-set [2] and harbored variants associated with sporadic DCM in our EWAS. MYBPC3 is an actin, myosin and titin interacting protein of the M-band of the sarcomere. Mutations in this gene are a major cause of hypertrophic cardiomyopathy and have also been reported in familial forms of DCM [35]. Coding variants in MYBPC3 may affect actin-myosin interaction [36] and concurrently interfere with the ubiquitin proteasome system and autophagy in humans and animal models [37]. Both mechanisms could account for the association of common SNVs in $M Y B P C 3$ with sporadic DCM.

Considered as a whole, both rare and common variants with elevated CADD scores in the cardiomyopathy gene-set were associated with sporadic DCM ( 33 Table) indicating that other loci than those found in this EWAS are involved in sporadic DCM, however identifying the responsible genes will require larger studies. 


\section{Proteostasis might be important for DCM}

Three of the DCM-associated genes, FLNC, TTN (through its kinase activity) and cardiac specific FHOD3 encode maintenance partners of sarcomere and sarcomere-related structures, including Z-disk or F-actin myofibrils [29,38,39], which are disorganized or degraded in experimental models of cardiomyopathy [40]. Moreover the cellular level of FLNC, FHOD3 and TTN kinase targets such as MuRF2, appears regulated by proteostasis mechanisms $[26,29,39]$. One of these mechanisms, BAG3-associated chaperone-assisted selective autophagy (CASA) is described as a central adaptation mechanism that responds to acute physical exercise and to repeated mechanical stimulation [41]. BAG3 inactivation also leads to Z-disk disruption in mice and fruit fly [26]. Based on the functional similarities (also pertaining to HSPB7 and MYBPC3) characterizing several of the DCM-associated genes identified in this study, we hypothesize that abnormal cardiomyocyte sarcomere maintenance and regulation of autophagy is a potential mechanism involved in DCM pathophysiology is. Further experimental exploration of this hypothesis may yield novel therapeutic targets for DCM.

\section{Limitations of this study}

This study has some limitations. The recruitment was focused on a priori homogeneous sets of patients and controls of European ancestry and outliers were excluded based on genomic data. We also conducted a meta-analysis which did not reveal any significant heterogeneity across populations. Despite these precautions, we cannot fully exclude undetected population stratification. In addition, given the rather low prevalence of DCM, we conducted exome-wide genotyping in all available patients and controls instead of using a two-step discovery/replication design. As a consequence, even if we provide a series of arguments supporting the identified genes as plausible candidates, independent studies would certainly further refine and extend our results. It is likely that a genome-wide tagging array not limited to exon regions would identify other DCM-associated variants and loci than those reported here. Finally, as our power analyses show, our EWAS had limited power to detect the collective effect of rare variants present in our data set at the gene level.

\section{Conclusion}

We identified 6 novel loci associated with sporadic DCM and confirmed two previously reported associations with variants located within the ZBTB17-HSPB7 and BAG3 genes. Fine mapping revealed that at the ZBTB17 locus HSPB7 is likely the implicated gene. The leadSNVs at all associated loci are common variants and conditioning on them reduced considerably the associations of other variants in the regions of interest with DCM. We provide evidence that 7 of the DCM-associated genes are very plausible candidates from a pathogenic perspective.

\section{Supporting information}

S1 Methods.

(DOCX)

S1 Fig. QQ Plot of association p-values.

(PDF)

S2 Fig. Manhattan and QQ plots by population.

(PDF) 
S3 Fig. Endogenous BAG3 interacts with GST-tagged HSPB7 in Hela cells. (TIF)

S1 Table.

(DOCX)

S2 Table. Number of DCM patients and controls.

(DOCX)

S3 Table. In Silico fine mapping at loci.

(DOCX)

S4 Table. SKAT analysis of variants in genes known to be associated with familial cardiomyopathy.

(DOCX)

S5 Table. Summary statistics-additive model.

S6 Table. Summary statistics-dominant model.

(TXT)

S7 Table. Association statistics comparison with and without the 90 FDCM cases.

(DOCX)

\section{Acknowledgments}

pEGFP-BAG3 construct was kindly provided by Dr S Takayama. We thank all patients and controls included in the studies.

\section{Author Contributions}

\section{Conceptualization: FC UE PC EV.}

Formal analysis: AB FC SG JL MMN JS.

Funding acquisition: EA FC PC SC GK PL VRZ CS EV.

Investigation: PB TC PC SC JPE UE SG HH HG CH RI XJ GK MK AK JK KM MM MMN DO C Proust SP C Perret JS K Stark K Strauch.

Resources: EA PB TC PC SC HH CH RI XJ MK PL KM.

Supervision: EA FC PC SC CH GK PL VRZ JS CS EV.

Writing - original draft: FC UE AK LT EV.

Writing - review \& editing: EA AB TC PC JPE SG HG CH XJ GK MK JK PL KM MMN VRZ CS K Strauch.

\section{References}

1. Hershberger RE, Hedges DJ, Morales A. Dilated cardiomyopathy: the complexity of a diverse genetic architecture. Nat Rev Cardiol. 2013; 10: 531-547. doi: 10.1038/nrcardio.2013.105 PMID: 23900355

2. McNally EM, Golbus JR, Puckelwartz MJ. Genetic mutations and mechanisms in dilated cardiomyopathy. J Clin Invest. 2013; 123: 19-26. doi: 10.1172/JCI62862 PMID: 23281406 
3. Villard E, Perret C, Gary F, Proust C, Dilanian G, Hengstenberg C, et al. A genome-wide association study identifies two loci associated with heart failure due to dilated cardiomyopathy. Eur Heart J. 2011; 32: 1065-1076. doi: 10.1093/eurheartj/ehr105 PMID: 21459883

4. Cappola TP, Li M, He J, Ky B, Gilmore J, Qu L, et al. Common variants in HSPB7 and FRMD4B associated with advanced heart failure. Circ Cardiovasc Genet. 2010; 3: 147-154. doi: 10.1161/ CIRCGENETICS.109.898395 PMID: 20124441

5. Cappola TP, Matkovich SJ, Wang W, van Booven D, Li M, Wang X, et al. Loss-of-function DNA sequence variant in the CLCNKA chloride channel implicates the cardio-renal axis in interindividual heart failure risk variation. Proc Natl Acad Sci U S A. 2011; 108: 2456-2461. doi: 10.1073/pnas. 1017494108 PMID: 21248228

6. Chang CC, Chow CC, Tellier LC, Vattikuti S, Purcell SM, Lee JJ. Second-generation PLINK: rising to the challenge of larger and richer datasets. GigaScience. 2015; 4: 7. doi: 10.1186/s13742-015-0047-8 PMID: 25722852

7. Yang J, Lee SH, Goddard ME, Visscher PM. Genome wide complex trait analysis (GCTA): methods, data analyses, and interpretations. Methods Mol Biol Clifton NJ. 2013; 1019: 215-236.

8. Ionita-Laza I, Lee S, Makarov V, Buxbaum JD, Lin X. Sequence Kernel Association Tests for the Combined Effect of Rare and Common Variants. Am J Hum Genet. 2013; 92: 841-853. doi: 10.1016/j.ajhg. 2013.04.015 PMID: 23684009

9. Howie BN, Donnelly P, Marchini J. A Flexible and Accurate Genotype Imputation Method for the Next Generation of Genome-Wide Association Studies. PLoS Genet. 2009; 5.

10. Kircher M, Witten DM, Jain P, O’Roak BJ, Cooper GM, Shendure J. A general framework for estimating the relative pathogenicity of human genetic variants. Nat Genet. 2014; 46: 310-315. doi: 10.1038/ng. 2892 PMID: 24487276

11. Consortium GTEx. The Genotype-Tissue Expression (GTEx) project. Nat Genet. 2013; 45: 580-585. doi: 10.1038/ng.2653 PMID: 23715323

12. Gerull B, Gramlich $M$, Atherton J, McNabb M, Trombitás K, Sasse-Klaassen S, et al. Mutations of TTN, encoding the giant muscle filament titin, cause familial dilated cardiomyopathy. Nat Genet. 2002; 30: 201-204. doi: 10.1038/ng815 PMID: 11788824

13. Wolf $C M$, Wang L, Alcalai R, Pizard A, Burgon PG, Ahmad F, et al. Lamin A/C haploinsufficiency causes dilated cardiomyopathy and apoptosis-triggered cardiac conduction system disease. J Mol Cell Cardiol. 2008; 44: 293-303. doi: 10.1016/j.yjmcc.2007.11.008 PMID: 18182166

14. Van Sligtenhorst I, Ding Z-M, Shi Z-Z, Read RW, Hansen G, Vogel P. Cardiomyopathy in a-kinase 3 (ALPK3)-deficient mice. Vet Pathol. 2012; 49: 131-141. doi: 10.1177/0300985811402841 PMID 21441111

15. Vos MJ, Zijlstra MP, Kanon B, van Waarde-Verhagen MAWH, Brunt ERP, Oosterveld-Hut HMJ, et al. HSPB7 is the most potent polyQ aggregation suppressor within the HSPB family of molecular chaperones. Hum Mol Genet. 2010; 19: 4677-4693. doi: 10.1093/hmg/ddq398 PMID: 20843828

16. Selcen D, Muntoni F, Burton BK, Pegoraro E, Sewry C, Bite AV, et al. Mutation in BAG3 causes severe dominant childhood muscular dystrophy. Ann Neurol. 2009; 65: 83-89. doi: 10.1002/ana.21553 PMID: 19085932

17. Jenkitkasemwong S, Wang C-Y, Mackenzie B, Knutson MD. Physiologic implications of metal-ion transport by ZIP14 and ZIP8. Biometals Int J Role Met lons Biol Biochem Med. 2012; 25: 643-655.

18. Li B, Tan Y, Sun W, Fu Y, Miao L, Cai L. The role of zinc in the prevention of diabetic cardiomyopathy and nephropathy. Toxicol Mech Methods. 2013; 23: 27-33. doi: 10.3109/15376516.2012.735277 PMID: 23039870

19. Ahmady E, Deeke SA, Rabaa S, Kouri L, Kenney L, Stewart AFR, et al. Identification of a novel muscle A-type lamin-interacting protein (MLIP). J Biol Chem. 2011; 286: 19702-19713. doi: 10.1074/jbc.M110. 165548 PMID: 21498514

20. Broers JLV, Ramaekers FCS, Bonne G, Yaou RB, Hutchison CJ. Nuclear lamins: laminopathies and their role in premature ageing. Physiol Rev. 2006; 86: 967-1008. doi: 10.1152/physrev.00047.2005 PMID: 16816143

21. Huang Z-P, Young Seok H, Zhou B, Chen J, Chen J-F, Tao Y, et al. CIP, a cardiac Isl1-interacting protein, represses cardiomyocyte hypertrophy. Circ Res. 2012; 110: 818-830. doi: 10.1161/ CIRCRESAHA.111.259663 PMID: 22343712

22. Huang Z-P, Kataoka M, Chen J, Wu G, Ding J, Nie M, et al. Cardiomyocyte-enriched protein CIP protects against pathophysiological stresses and regulates cardiac homeostasis. J Clin Invest. 2015; 125: 4122-4134. doi: 10.1172/JCI82423 PMID: 26436652 
23. Valdés-Mas R, Gutiérrez-Fernández A, Gómez J, Coto E, Astudillo A, Puente DA, et al. Mutations in filamin $C$ cause a new form of familial hypertrophic cardiomyopathy. Nat Commun. 2014; 5: 5326. doi: 10.1038/ncomms6326 PMID: 25351925

24. Kley RA, van der Ven PFM, Olivé M, Höhfeld J, Goldfarb LG, Fürst DO, et al. Impairment of protein degradation in myofibrillar myopathy caused by FLNC/filamin C mutations. Autophagy. 2013; 9: 422-423. doi: 10.4161/auto.22921 PMID: 23238331

25. Selcen D. Myofibrillar myopathies. Neuromuscul Disord NMD. 2011; 21: 161-171. doi: 10.1016/j.nmd 2010.12.007 PMID: 21256014

26. Arndt V, Dick N, Tawo R, Dreiseidler M, Wenzel D, Hesse M, et al. Chaperone-assisted selective autophagy is essential for muscle maintenance. Curr Biol CB. 2010; 20: 143-148. doi: 10.1016/j.cub.2009. 11.022 PMID: 20060297

27. Juo L-Y, Liao W-C, Shih Y-L, Yang B-Y, Liu A-B, Yan Y-T. HSPB7 interacts with dimerized FLNC and its absence results in progressive myopathy in skeletal muscles. J Cell Sci. 2016; 129: 1661-1670. doi: 10.1242/jcs.179887 PMID: 26929074

28. Kan-O M, Takeya R, Abe T, Kitajima N, Nishida M, Tominaga R, et al. Mammalian formin Fhod3 plays an essential role in cardiogenesis by organizing myofibrillogenesis. Biol Open. 2012; 1: 889-896. doi: 10.1242/bio.20121370 PMID: 23213483

29. Iskratsch T, Lange S, Dwyer J, Kho AL, dos Remedios C, Ehler E. Formin follows function: a musclespecific isoform of FHOD3 is regulated by CK2 phosphorylation and promotes myofibril maintenance. $J$ Cell Biol. 2010; 191: 1159-1172. doi: 10.1083/jcb.201005060 PMID: 21149568

30. Xu Y, Moseley JB, Sagot I, Poy F, Pellman D, Goode BL, et al. Crystal structures of a Formin Homology-2 domain reveal a tethered dimer architecture. Cell. 2004; 116: 711-723. PMID: 15006353

31. Arimura T, Takeya R, Ishikawa T, Yamano T, Matsuo A, Tatsumi T, et al. Dilated cardiomyopathy-associated FHOD3 variant impairs the ability to induce activation of transcription factor serum response factor. Circ J Off J Jpn Circ Soc. 2013; 77: 2990-2996.

32. Hosoda T, Monzen K, Hiroi Y, Oka T, Takimoto E, Yazaki Y, et al. A novel myocyte-specific gene Midori promotes the differentiation of P19CL6 cells into cardiomyocytes. J Biol Chem. 2001; 276: 3597835989. doi: 10.1074/jbc.M100485200 PMID: 11418590

33. Snyder M, Huang X-Y, Zhang JJ. Stat3 directly controls the expression of Tbx5, Nkx2.5, and GATA4 and is essential for cardiomyocyte differentiation of P19CL6 cells. J Biol Chem. 2010; 285: 2363923646. doi: 10.1074/jbc.M110.101063 PMID: 20522556

34. Almomani R, Verhagen JMA, Herkert JC, Brosens E, van Spaendonck-Zwarts KY, Asimaki A, et al. Biallelic Truncating Mutations in ALPK3 Cause Severe Pediatric Cardiomyopathy. J Am Coll Cardiol. 2016; 67: 515-525. doi: 10.1016/j.jacc.2015.10.093 PMID: 26846950

35. Hershberger RE, Norton N, Morales A, Li D, Siegfried JD, Gonzalez-Quintana J. Coding Sequence Rare Variants Identified in MYBPC3, MYH6, TPM1, TNNC1, and TNNI3 From 312 Patients With Familial or Idiopathic Dilated Cardiomyopathy. Circ Cardiovasc Genet. 2010; 3: 155-161. doi: 10.1161/ CIRCGENETICS.109.912345 PMID: 20215591

36. Previs MJ, Beck Previs S, Gulick J, Robbins J, Warshaw DM. Molecular mechanics of cardiac myosinbinding protein $C$ in native thick filaments. Science. 2012; 337: 1215-1218. doi: 10.1126/science. 1223602 PMID: 22923435

37. Schlossarek S, Englmann DR, Sultan KR, Sauer M, Eschenhagen T, Carrier L. Defective proteolytic systems in Mybpc3-targeted mice with cardiac hypertrophy. Basic Res Cardiol. 2012; 107: 235. doi: 10. 1007/s00395-011-0235-3 PMID: 22189562

38. van der Ven PF, Obermann WM, Lemke B, Gautel M, Weber K, Fürst DO. Characterization of muscle filamin isoforms suggests a possible role of gamma-filamin/ABP-L in sarcomeric Z-disc formation. Cell Motil Cytoskeleton. 2000; 45: 149-162. doi: 10.1002/(SICI)1097-0169(200002)45:2<149::AID-CM6>3. 0.CO;2-G PMID: 10658210

39. Lange S, Xiang F, Yakovenko A, Vihola A, Hackman P, Rostkova E, et al. The kinase domain of titin controls muscle gene expression and protein turnover. Science. 2005; 308: 1599-1603. doi: 10.1126/ science.1110463 PMID: 15802564

40. Fujita M, Mitsuhashi H, Isogai S, Nakata T, Kawakami A, Nonaka I, et al. Filamin C plays an essential role in the maintenance of the structural integrity of cardiac and skeletal muscles, revealed by the medaka mutant zacro. Dev Biol. 2012; 361: 79-89. doi: 10.1016/j.ydbio.2011.10.008 PMID: 22020047

41. Ulbricht A, Gehlert S, Leciejewski B, Schiffer T, Bloch W, Höhfeld J. Induction and adaptation of chaperone-assisted selective autophagy CASA in response to resistance exercise in human skeletal muscle. Autophagy. 2015; 11: 538-546. doi: 10.1080/15548627.2015.1017186 PMID: 25714469 